\title{
EL ITINERARIO DE LOS JURISTAS: UN VIAJE A BOLONIA EN 1838
}

\author{
Salvador Claramunt
}

Las relaciones hispano-italianas siempre han tenido una gran porosidad y vitalidad desde la época del Imperio romano, e incluso en los momentos más difíciles de la Alta Edad Media. Cataluña, por su situación geográfica y, más tarde, por la política mediterránea de sus soberanos, que no hicieron más que encauzar los impulsos comerciales de la burguesía del Principado, ocupará un lugar privilegiado en las relaciones con la península itálica.

Con este modesto trabajo, en el que se pormenoriza el viaje de un joven estudiante de Cervera a Bolonia en la primera mitad del siglo XIX, quiero colaborar en esta publicación de homenaje al profesor Federico Udina Martorell, $\tan$ ferviente continuador de las relaciones hispano-italianas en sus más diversas facetas.

Desde que, a finales del siglo XII, Bolonia y París se constituyeron en los dos centros culturales más importantes en donde se impartían respectivamente los conocimientos más avanzados de la época de derecho y de teología, numerosos jóvenes y no tan jóvenes ansiosos de aumentar sus conocimientos sobre dichas materias acudirán desde todos los rincones de la Europa cristiana a ambas ciudades.

Bolonia recibirá multitud de estudiosos que querían alcanzar la licentia docendi en su famosa Facultad de Jurisprudencia. Religio- 
sos, políticos, gentes del ámbito mercantil atravesarán Europa para acudir a la ciudad emiliana y escuchar las lecciones magistrales de Irnerio y más tarde de Accursio, y de los demás glosadores. De entre los estudiantes que allí acudían, los hispánicos eran un grupo muy notable de entre los ultramontanos, y de los hispánicos era a su vez muy considerable el número de catalanes que había en Bolonia ya en la primera mitad del siglo XII, tanto que de las dieciocho naciones o corporaciones de estudiantes cuyo conjunto formaba la universidad, una era la de los catalanes. En 1211, Ramón de Penyafort debió de llegar a Bolonia para estudiar derecho romano, siendo promovido a la función docente en el Studium boloñés al cabo de unos años 1 ; también por aquella época era profesor de la misma un jurisconsulto leridano llamado Pons, según consta en la lista de profesores de Bolonia de 1213. El caso de Ramón de Penyafort no fue ni único ni casual, pues un numeroso grupo de súbditos de la Corona catalano-aragonesa acudía cada año para iniciar su etapa de estudios jurídicos en Bolonia, dándose la circunstancia de que los hispanos son los primeros profesores de más allá de los Alpes que enseñaron en las cátedras del Alma Studiorum Mater, donde estuvieron rodeados de un numeroso grupo de estudiantes hispánicos que los admiraban con el fervor de los néfitos.

Pero prescindiendo del hecho de la existencia de la famosa universidad boloñesa, las relaciones permanentes entre los reinos hispánicos y Bolonia comenzaron en el siglo XII, al producirse una circunstancia peculiar debida a un error de lectura en una indicación de las reliquias de un santo sepultado en la iglesia del monasterio de San Esteban de Bolonia, recogido después este error en un manuscrito en 1141 en donde se narra el reconocimiento de las reliquias de dicha basílica realizada en aquel año. Se trataba nada menos que del cuerpo de san Isidoro de Sevilla².

1 Fernando Valls Y TABerner, San Ramón de Penyafort, Nueva Colección Labor, Barcelona, 1979.

2 A. CAsale, Nuova Gierusalemme detta la Sacra Basilica di San Stefano di Bologna, Bologna, 1627. 
...similiter et corpus beatissimi Ysidori confessoris Christi et loculentissimi dactoris in te requiescit. Qui cum rediret a Roma apud hanc urbem hospitatus ac gravia molestia corporis detentus, permitente Deo, ibique vitam finivit et illic ab hortodoxis viris cum gaudio plebis sepultus est.

Esta afirmación fue recogida con certeza por casi todos los cronistas e historiadores boloñeses, hasta que en el siglo XIII comenzó a ponerse en duda, y se pensó con razón que se había tratado de un grave error de interpretación (san Isidoro de Sevilla por Sicilia) ${ }^{3}$, surgido sin consultar para nada la historiografía hispánica sobre el traslado del cuerpo de san Isidoro a León.

Pero a pesar de todo, la fiesta de san Isidoro de Sevilla era celebrada por la iglesia boloñesa con especial solemnidad desde el siglo XI, y la presencia del cuerpo del santo hispano debió de atraer a numerosos compatriotas desde el primer momento, más si tenemos en cuenta que los juristas siempre vieron en san Isidoro un gran maestro, no sólo por sus obras auténticas, sino especialmente por la falsa atribución de la colección, atribuida a él, pero no redactada por él, de los decretales pseudoisidorianos del siglo IX.

El caso es que mucho antes de que finalizase el siglo XII, tenemos constancia de diversos profesores hispanos 4 , como un tal Melendo, que habría enseñado en Bolonia hacia 1168; Petrus hispanicus en 1180; Juan Diano Aragonés, Lorenzo Ispano, Bernardo Compostellano sénior y júnior, Juan Petesella, Juan García, Martín de Zamora, otro Petrus hispanus, Vicente Ispano, Petrus de Lérida, Álvaro Pelagio, amén de los antes citados Pons de Lérida y Ramón de Penyafort.

Otro momento crucial en las relaciones de Bolonia con la península ibérica, será la llegada a dicha ciudad de santo Domingo de Guzmán 5 , en la cual moriría años después. Sabemos que, en 1220 ,

${ }^{3}$ G.B. MeLLoNi, Auti e Memorie degli Vomini lllustri in Santità nati o mort in Bologna, Classe I, vol. I, Bologna, 1786, pp. 521 y ss.

${ }^{4}$ C. MEsIN, «Gli spagnoli a Bologna» pp. 41-71 en Studia Albornotiana, XII, dedicado a $\propto$ EI cardenal Albornoz y el colegio de España», Bolonia, 1972.

5 Gerardi DE FRANCHE, Vita Fratum Ordinis Pradicatorum, Louvain, 1896. p. 83 . 
Domingo de Guzmán convocó un capítulo de su orden en la ciudad y se interesó especialmente por los estudiantes hispanos. Murío en 1221, y su sepulcro se convirtió en un lugar de peregrinación de todo hispano que recorriese el norte de Italia.

En el primer cuarto del siglo XIV destacó un Jaime de Valencía que fue decapitado en 1321 por haber raptado a Constanza Zagnoni, hija de uno de los más poderosos ciudadanos de Bolonia, hecho que ocasionó la marcha de muchos estudiantes y doctores, primero a Imola y después de Siena ${ }^{6}$.

Pero es con la presencia del cardenal Gil de Albornoz como legado pontificio en Italia, cuando a partir de 1360 se intensificaron aún más las relaciones entre la península ibérica y la ciudad emiliana, culminando con la fundación del Colegio de España en 1364, como voluntad postrera del cadenal Albornoz, para acoger a los estudiantes de los distintos reinos ibéricos cristianos. En 1368 comenzaron a ser admitidos los primeros colegiales, y en aquel mismo año son ya 27 , cifra que se elevará a 582 estudiantes colegiales de la institución albornociana antes de iniciarse el siglo XVI?

No tratamos de demostrar en este trabajo la importacia que tuvo Bolonia para el estudioso de leyes, ni menos cuantificar cuántos estudiantes fueron a dicha universidad, Solamente queremos dar a conocer un itinerario de viaje desde Cataluña a Italia, y más concretamente a Bolonia. Las noticias medievales y de época moderna sobre los itinerarios efectuados por los clérigos, estudiantes, hombres de leyes, etc. que fueron a Bolonia son numerosas pero incompletas; sabemos de ciudades o poblaciones que eran etapa, y muchas son las referencias a que el viaje se realizaba de modo mixto por tierra y por mar.

En una visita efectuada a la ciudad de Cervera, la archivera $D^{a}$ Francina Solsona tuvo la gentileza de ponerme en contacto con la familia Nuix, que me mostró el diario de un viaje efectuado por

6 F. Fil.IPPINI, $\alpha$ L'Esodo degli studenti da Bologna nel 1321 w en Studi e Memoria per la sotira della Universita di Bologna, VI, Bologna, 1921, pp. 107 y ss.

7 Antonio PÉREz MARTíN, «ProlesAggidirana», I, Introducción. Studia Albornotiana, Bolonia, Xxxi, 1979. 
un antepasado suyo, D. Joaquín de Nuix y de Ferrer, en 1838, de Ceret a Bolonia, conservado en el archivo de la familia; el detallismo con que el viaje es narrado y el itinierario llamaron mi atención, pues diversas veces había realizado el mismo recorrido y los puntos de coincidencia 130 años después eran más bien escasos una vez efectuado el ineludible paso por el sur de Francia.

Joaquín de Nuix y de Ferrer, autor del diario que nos permitirá trazar el itinerario a Bolonia, perteneció a una ilustre familia de Cervera, hijo de Luis María de Nuix y de Pontich, y nieto de Francisco de Nuix y de Perpinyà; hermano de Juan de Nuix y de Perpinyà ${ }^{8}$, escritor y eclesiástico. Éste último fue el más ilustre personaje de la familia Nuix, que había estudiado teología en Barcelona e ingresado en la Compañía de Jesús. Al ser expulsada ésta en 1867, se estableció en Ferrara, en donde publicó Riflessioni imparziali sopra l' umanità degli spagnoli nell' Inde en 1770, en donde defendió la obra colonizadora en América.

El abuelo de nuestro personaje fue el primer Barón de Perpiñán. Nuestro personaje, Joaquín de Nuix y de Ferrer, nació en Cervera el 16 de febrero de 1816, cursó estudios de gramática latina en los dominicos de Gerona, más tarde leyes en Cervera y finalmente se doctoró en ambos derechos (civil y canónico) en Bolonia; fue nombrado promotor fiscal en 1844 , cargo que ejerció en la propia Cervera; casó con Joaquina de Espona y de Espona, y tuvo un hijo llamado Antonio de Nuix y de Espona, diputado carlista por Cervera en 1883. Joaquín de Nuix y de Ferrer murió el 15 de setiembre de 1865 de «cólera morbo asiático», según en el archivo de la casa de Nuix en Cervera.

El itinerario descrito por Joaquín de Nuix y de Ferrer, a los 22 años, cuando acude a doctorarse a Bolonia, no debió de variar del de tantos y tantos estudiantes que habían ido a Bolonia anteriormente.

Detallamos a continuación el recorrido efectuado día a día del 21 de noviembre de 1838 , fecha en que sale de Ceret, hasta el 24 de enero de 1839 , en que regresa a la misma población de partida. Hay

8 José M. Razquin Jene, Noticia de la vida y obra del P. Juan de Nuix y Perpinyá, Instituto de Estudios Ilerdenses, Lérida 1971. 
que hacer constar que el diario del viaje está repleto de anécdotas y de explicaciones muy partiuclares de cada población que atraviesa, así como de los gastos efectuados. Hemos prescindido de todos estos detalles para centrarnos en lo que propiamente nos interesa, el itinerario del viaje.

\section{1 de noviembre}

Salida a las 14,15 horas de Ceret. A las 15 horas llegada a Le Boulou, a orillas del río Tech y cambio de caballos.

Llegada a Perpiñán a las 17,15 horas. Duerme en casa de un primo.

\section{2 de noviembre}

Permanece todo el dia en Perpiñán para hacer diligencias y sacar el pasaporte hasta Marsella. Salida a las 20,15 horas con dirección a Salses.

\section{3 de novimebre}

A las 2 horas de la madrugada llegada a Sigean y cambio de caballos. A las 4 horas llegada a Narbona, una hora de parada.

A las 5 horas salida hacia Nissan y llegada a Béziers a las 8,45 horas, en donde se efectúa una parada para el desayuno, de una hora. Salida de Béziers a las 9,45 y llegada a las 12 horas a Agde, cambio de tiro y salida hacia Marseillan, a donde se llega a las 12,45 horas.

Llegada a Mèze a las 14,45 horas y cambio de tiro. Después de pasar dos o tres pueblos insignificantes entre subidas y bajadas se llega a Montpellier a las 18,30 horas. Cena y alojamiento.

\section{4 de noviembre}

Mañana libre en Montpellier, en donde visita lo más importante de la ciudad.

A las 11,30 horas se toma de nuevo la diligencia con dirección a Nîmes. A las 13,30 horas se llega a Lunel y se cambian los caballos. Llegada a las 17 horas a Nîmes. Cambio de diligencia y salida a las 18 horas para Aix-en-Provence. Se atraviesa el Ródano por 
Beaucaire y Tarascon. A las 21 horas se pasa por St. Rémy en dirección a Orgon.

\section{5 de noviembre}

Durante la madrugada se pasa por Lambesc y se llega a St. Cannat a las 8 de la mañana.

La llegada a Aix-en-Provence se efectúa a las 10,30 horas. Pasa todo el dia en Aix en casa de un tío y otros familiares, que le muestran la ciudad.

\section{6 de noviembre}

Salida de Aix a las 5 horas y llegada a Marsella a las 8,45 . Se arregla el pasaporte y visita la ciudad el resto del día.

\section{7 de noviembre}

Salida de Marsella a las 14 horas con dirección a Aix, a donde se llega a las 18 horas.

\section{8 de noviembre}

Salida de Aix a las 16 horas con dirección a Niza. A las 22 horas se llega a Brignoles y a las 24 horas a Le Luc.

29 de noviembre

A las 3 de la madrugada llegada a Draguignan. A las 6,30 horas a Fréjus, y al amanecer se pasa el macizo del Esterel. A las 10,30 se llega a Cannes. A las 11,45 a Antibes, última ciudad de Francia en esta frontera. Se llega al río Var, que divide Francia del Piamonte, a las 14,30 y se realizan las diligencias fronterizas. Problemas para entrar en Piamonte que no se solucionaron hasta las 18 horas, llegando a las 19 horas a Niza, donde se hospedó en el Colegio de los jesuitas.

30 de noviembre y $1,2,3$ y 4 de diciembre

Están en Niza visitando a unos tíos y primos.

5 de diciembre

A las 17,30 horas embarca en el vapor toscano El Etrusco hacia Génova. 


\section{6 de diciembre}

Llegada a las 8,30 horas al puerto de Génova. Recorre por la mañana Génova acompañado por unos jesuitas. A las 19 horas el barco zarpa de nuevo, con rumbo a Livorno.

\section{7 de diciembre}

Llegada a las 8,30 horas al puerto de Livorno. Gestiona el pasaporte y lo hace visar.

\section{8 de diciembre}

A las 7,30 horas sale de Livorno en la diligencia que va a Florencia. El camino que conduce de Livorno a Florencia, afirma, es el más hermoso que jamás haya visto; es el jardín de Italia. Se bordea el Arno y a las 11 horas se llega a Fornaceta, en donde se cambian los caballos. A las 11,35 se llega a Empoli, y hay dos horas libres para cambiar de tiro y comer. A las 18 horas se llega a Florencia.

\section{9 de diciembre}

Pasa todo el día en Florencia, aunque hace constar que vio muy pocas cosas por estar casi todo cerrado, al ser domingo.

\section{0 de diciembre}

A las 6,30 se sale de Florencia, iniciando la subida de los Apeninos. Todo el camino no son más que subidas y bajadas hasta dos horas antes de llegar a Bolonia, según nos cuenta el protagonista en su diario.

A las $15 \mathrm{~h}$. se llega a la gran muralla que ha hecho construir el gran duque reinante Leopoldo II (1824-1859) para evitar el mal paso que hay a causa de las fuertes corrientes de viento, que hacen volcar los carruajes (así consta en el diario de viaje). A las 18 horas se llega a Seca, aduana papal, pero antes se encuentra la aduana del Gran Ducado de Módena. En este trozo la niebla es muy densa; se cena y se pasa la noche.

\section{1 de diciembre}

Salida de Seca a las 7,30 horas, llegada a Pianoro a las 12 horas, comida y descanso de 2 horas. A las 14,15 horas salida de Pianoro y entrada en la llanura del Boloñés. A las 16,30 horas llegada a Bolonia. 


\section{Del 12 al 22 de diciembre}

Está en Bolonia, en donde tomó el grado de doctor el dia 20.

\section{8 de diciembre}

Salida de Bolonia a las 6,30 horas. Llegada a Loiano a las 10,45 horas, en donde se come. A las 12 horas salida hacia Covigliano, a donde se llega a las 21,15 horas, cena y alojamiento en dicha población, en el corazón de los Apeninos.

\section{9 de diciembre}

Salida a las 5,30 horas, con viento y nieve, hacia Tagliaferro, en donde se descansó hora y media para comer (de 11 a 12,30 horas). A las, 12,30 horas salida hacia Florencia, a donde se llega a las 15,30 horas.

30 y 31 de diciembre

Permanece todo el día en Florencia, visitándola de nuevo.

\section{1 de enero de 1893}

Salida a las 7,30 horas con dirección a Livorno. A las 10,30 horas se llega a Empoli, en donde se detienen media hora para cambiar de carruaje y caballos. A las 13,30 horas llegada a Pontedera, cambio de caballos y salida para Pisa, a donde se llega a las 14,30, visita la catedral con su famoso companario y a las 16 horas salía hacia Livorno, ciudad a la que llega a las 18 horas.

Días 2 y 3 de enero

Permanece en Livorno en espera del vapor que lo traslada a Génova.

\section{4 de enero.}

Por la tarde embarca en el vapor El Rómulo hacia Génova.

\section{5 de enero}

Llegada a las 4 horas de la madrugada a Génova. A las 8 horas desembarca y a las 16 horas tomaba la diligencia-correo hacia Niza.

6 de enero

Llega a Niza a las 20,30 horas, en donde encontró de nuevo a su tío. 


\section{Del 7 al 15 de enero}

Permanece en Niza con sus familiares.

\section{5 de enero}

Sale hacia Toulon, sin especificar la hora exacta.

\section{6 de enero}

Se supone que está de viaje camino de Toulon.

\section{7 de enero}

Llega a Toulon a las 3 horas de la madrugada. Visita durante todo el día a unos amigos y el arsenal. Por la noche parte para Marsella.

\section{8 de enero}

Llega a Marsella a las 4 horas de la madrugada.

\section{9 de enero}

Salida de Marsella a las 5 horas con dirección a Arles.

20 de enero

Visita los principales monumentos de Arles y por la tarde parte hacia Nîmes y Montpellier en la diligencia.

\section{1 de enero}

Se llega a Montpellier a las 3 horas de la madrugada. Y a las 17 horas partía hacia Perpiñán.

\section{2 de enero}

Llegada a la capital del Rosellón a las 17 horas.

\section{3 de enero}

Permanece todo el día en Perpiñán.

\section{4 de enero}

A las 8 horas llegada a Ceret, cansado de las numerosas fatigas de tan largo viaje. Empieza a redactar el diario del viaje acabado de realizar.

$$
* * *
$$

De lo acabado de exponer se deduce que la ruta normal en 1838 era más o menos la misma que en la Edad Media a su paso por Perpiñán, Salses, Sigean, Narbona, Béziers, Agde, Marseillan, Mèze, 
Montpellier, Lunel y Nîmes. Este trayecto, de unos 250 kilómetros, se efectúa en casi tres días, y recorre el mismo itinerario de la vía romana que unía Barcelona con Nîmes, pasando por las antiguas ciudades romanas de Narbo, Betera y la colonia griega de Agata.

De Nîmes a Aix-en-Provence se utiliza una ruta que pasa por Beaucaire, Tarascon, St. Rémy, Orgon, Lambesc, cruzando el R6dano a la altura de Beaucaire por un puente colgante de cinco arcos y de una longitud de $440 \mathrm{~m}$. Con este recorrido se ha abandonado el paso obligatorio del Ródano por el puente de Arles, último antes de la desembocadura del río, y la ruta de Salon, seguida en la Edad Media. La distancia entre los dos trayectos vienen a ser más o menos equivalente, con una diferencia de un kilómetro menos por la ruta moderna.

La ida a Marsella de nuestro eprsonaje queda justificada por cuestiones burocráticas de poner en regla el pasaporte, ya que se regresa de nuevo a Aix. Pero en la Edad Media hay numerosos testimonios que confirman a Marsella como puerto de embarque hacia Italia.

De Aix la ruta sigue a Brignoles, Le Luc, Draguignan, Fréjus, Cannes, Antibes, Niza; el recorrido es similar a la carretera general actual que une Aix con Fréjus, con la única excepción de Draguignan, población que queda hoy apartada unos 11 kilómetros de la ruta general. Hay que resaltar la lentitud con que se atraviesa el macizo del Esterel y la llegada a la frontera con el reino de Piamonte sobre el río Var; en total, unos 209 kilómetros.

En Niza se embarca hacia Livomo. Esta utilización del transporte marítimo no es casual, y puede ser constatado en los viajes utilizados desde el siglo XIV por los numerosos estudiantes que iban a Bolonia; su finalidad era evitar el paso de los Apeninos Ligures y sobre todo el tramo de la vía Aurelia, que va de Génova hasta la Spezia, con el pesadísimo puerto de Bracco.

De Livorno a Florencia se sigue el curso del rio Arno sin mayor dificultad, y después de la capital de la Toscana a Bolonia se atraviesan los apeninos por los puertos de la Futa y de Raticosa. El paso de los Apeninos, que hoy en día se efectúa gracias a la autopista en una hora, necesitaba, en pleno siglo XIX, de un día y medio, con alojamiento incluido en una población en el corazón de los Apeni- 
nos como es Covigliano, duración que se duplicaba en épocas anteriores cuando todavía no se contaba con la celeridad de las diligencias decimonónicas.

Puede extrañar hoy que se prefiriera ir a Bolonia por el sur en vez de hacerlo por el norte, vía valle del Po, pero lo que se intenta en el siglo XIX, y con más motivo en los siglos anteriores, era evitar el peligroso paso de los Alpes, sobre todo en invierno, en que permanecían largo tiempo bloqueados por la nieve; mientras que los Apeninos, a pesar de las dificultades, siempre serán menos difíciles de atravesar debido a su menor altitud.

El regreso se efectúa de nuevo vía Florencia, y en Livorno se embarca hacia Génova; esta vez se hace por tierra el recorrido de Génova a Niza, lo que confirma de nuevo que el verdadero obstáculo eran los Apeninos Ligures del puerto de Bracco.

La alternativa a este itinerario en los siglos XIV y XV era la eliminación del recorrido marítimo y alcanzar el valle del Po atravesando los Alpes por el Col de Tenda y por Cuneo, Asti y Alessandria, llegar a Parma y desde allí a Bolonia. A pesar de todo, lo más corriente será el itinerario mixto tierra-mar, que tendrá en los puertos de la Spezia y sobre todo Livorno el paso casi obligado desde Marsella, Niza, Savona o Génova. 


\section{APÉNDICE}

Se incluyen a continuación las hojas del texto original en que se explica el recorrido Florencia-Bolonia-Florencia, así como los gastos habidos durante la corta estancia en Bolonia.

Todo el diario del viaje está escrito en un castellano con numerosos galicismos, y lleno de observaciones personales muy expresivas de la mentalidad del estudiante de Cervera.

$$
* * *
$$

Florencia, 10 de diciembre.

Dejo la descripción de Florencia para cuando vuelva a esta ciudad pués ho ne podido ver casi nada de ella, por ser Domingo en que todo estaba cerrado.

\section{GASTO}

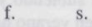

Leche por la mañana

Al garçon del hotel

Llevar el equipaje

15

Comida en el camino

Suma

$\begin{array}{r} \\ \\ \\ \\ \\ 2 \quad 15 \\ 15 \\ 15 \\ \hline\end{array}$

4

3

Bolonia, dia 11 de diciembre.

Salí de Florencia, ayer por la mañana a las seis y media y al instante empezamos la subida a los Apeninos.

La vista es hermosa pués se descubre toda la llanura desde Florencia hasta Liorna y el camino a pesar de ser montañoso es bonito por ser todo cultivado y por la multitud de árboles fructiferos y silvestres que hay. A las once de la mañana llegamos a Mascaras en donde comimos, haciendo de detención una hora.

Los Apeninos no son unas montañas, como me creía, áridas y cubiertas de peñascos; son, por el contrario, unos montes fértiles, cubiertos de árboles productivos y de mieses; únicamente los picos más elevados están incultos. 
A las trés de la tarde encontramos la gran Muralla que ha hecho construir el Gran Duque reinante para obviar el mal paso que hay a causa de las fuertes corrientes de viento, tan frecuentes en aquel punto, las que ocasionaban grandes desgracias, volcando los carruajes. Empiezan a encontrarse allí unos grandes palos que sirven para indicar el camino cuando está cubierto de nieve. Nosotros no la hemos encontrado sino en la montaña más elevada y en poca cantidad. Todo el camino no son más que subidas y bajadas hasta dos horas antes de llegar a Bolonia. A las seis de la tarde llegamos a Seca, donde está la Aduana del Papa y el principio de sus Estados. Pero antes, se encuentra la del Ducado de Modena en la que no nos registraron el equipaje. Hay también cerca de esta última, un pequeño volcán, el que no se vé sino de noche, más a pesar de eso no pude verlo pués había una niebla muy espesa. Allí cenamos y pasamos la noche volviendo a salir a la mañana siguiente, a las siete y media. Continuamente subiendo y bajando en medio de aquellas fértiles montañas, que en verano han de ser una vista magnífica, llegamos a las doce y cuarto a Pianora, población que está cuasi al pié de los Apeninos. Allí hemos comido haciendo de detención, dos horas.

A las dos y cuarto, volvimos a salir descubriendo yá la magnifica llanura del Bolonés, cuya vista se pierde a través de sus fértiles campos.

Por fin he llegado a Bolonia a las cuatro y media de la tarde.

Nota: Se ha de advertir que debe irse con mucho cuidado con los Veturinos o conductores de carruajes de alquilér. Piden siempre el doble del precio y, por la menor tontería, piden las agujetas o buena mano. Es preciso hacerles, antes los pactos: no pagarles antes. $\mathrm{Ha}-$ cer un escrito en que consten todos los pactos, es decir: la cena, el fuego y la cama del mesón en que se duerme y que, uno, no quiere entenderse en nada con criados o faquinis, que aqui llaman. Con todo, siempre se ha de dar algo, al criado del mesón, los que llevan el equipaje, etc. 
f. $s$.

Almuerzo

1

Comida

2

Al guarda para el Pasaporte

Llevar el equipaje en Bolonia

Regalo al Veturino o conductor

Suma

5

GASTO TOTAL DEL VIAJE DE NIZA A BOLONIA

16615

Bolonia, 12.

\section{GASTO}

f. $s$.

Paño para una casaca y un pantalón

70

1

Carta franquear

Suma

Día 17.

Carta de permanencia

Grado de Licenciado y Doctor

Sombrero

2

Guantes

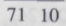

10

Suma

3135

Dia 19.

Regado al bedel de la Universidad

Tabaco

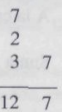

Suma

Correo 
Dia 20.

Tomé el grado.

Dia 21.

Para ir a ver N.S. de S. Luca y el Cementerio 210

Hechuras de la casaca y pantalón

Suma

2710

Dia 22.

Para ver las salas de la Universidad y la Torre. $\quad 1 \quad 18$

Certificado en la Universidad

215

Componer el relox

112

Tarjetas

Suma

f. s.

Dia 23.

Lavar la ropa

$1 \quad 10$

Dia 24.

Ir a ver las pinturas y la Sala de Armas

Dia 27.

Papel

Cartas

5

Pasaporte

14

Misa

215

Gasto de 17 dias en Bolonia, a 2 f. 5

Regalo (y el grado)

$38 \quad 5$

A la criada

5

Suma 


\section{CONTINUACIÓN DE MI VIAJE}

Florencia, 29 de diciembre de 1838.

Salí de Bolonia a las seis y media de la mañana del dia 28 y llegamos a Lojano a las diez y tres cuartos, en donde comimos, habiendo vuelto a salir a las doce. Hemos continuado, sin parar hasta Covigliano, en donde pasamos la noche. Llegamos allí a las nueve y cuarto. A pesar de estar todo el camino de Bolonia cubierto de nieve y de una espesa niebla, hemos podido observar perfectamente el pequeño volcán, que está a una media legua, distante de Covigliano, viniendo de Bolonia, a la izquierda, distante de la carretera un cuarto de hora, en medio de una montañita según lo que pude observar en aquella hora de noche. Desprende una luz colorada y muy viva, pareciendo un pequeño fuego encendido en aquel punto. El mal camino y el estar cubierto de nieve me ha impedido de ir allí cerca, pués lo deseaba.

A las cinco y media de esta mañana hemos vuelto a salir con viento y nieve la que hemos encontrado hasta cerca de Tagliaferro en donde, después de habernos detenido hora y media para comer, desde las once hasta las doce y media, hemos vuelto a salir, llegando a ésta a las tres y cuarto de la tarde.

He venido a parar a la Pensión de S. Louis que tienen la ventaja de ser céntrica, ser franceses y barata.

He tenido que tomar un carruaje de alquiler por ser la diligencia o correo tomada anticipadamente, por muchos dias. Este ha sido mas caro a causa del mal camino y gran cantidad de nive. El precio ordinario, en buén tienpo es $20 \mathrm{f}$., comprendida la buena mano, la carne, cama y fuego.

Permanecí en Bolonia diez y seis dias; los nueve primeros los dedique al estudio y los restantes, después de haberme graduado, en recorrer lo más notable que ofrecia la ciudad, con mis dos amigos 
de los que no puedo hacer más que elogios. Acompañáronme a varias casas, en las que fuí muy bién recibido y, una noche a la reunión más brillante de Bolonia en la que pensé varias veces con papá, pués se hubiera complacido en oir el más célebre pianista de la Europa.

Separeme de mis amigos el dia 28, a las cinco de la mañana en que partí con dirección a Florencia.

Es Bolonia una ciudad bastante hermosa, rica y la segunda de los Estados Pontificios. Tiene pocas calles derechas y ninguna espaciosa. Casi todas están adornadas de porticos en ambos lados irregulares en su forma, muy cómodos, a la verdad, para sus habitantes, pero que dan a la ciudad un aspecto triste y desierto.

Las Iglesias de Bolonia son numerosas y contienen cada una de ellas una multitud de objetos preciosos. La Catedral, la grande Iglesia de S. Petronio, S. Domingo y la de los P.P. Servitas, son dignas, cada una de ellas por su estilo, de atención.

Cerca de la puerta de Ravegnana, se elevan dos torres cuadradas admirables por su inclinación. La mas elevada, de 307 piés, tiene sobre 5 , de inclinación la otra es mas baja pero más inclinada.

La Universidad no presenta nada en el exterior, ni su interior puede compararse con la Universidad de Cervera. Muchas de sus salas están ocupadas por los gabinetes de Física, Anatomia, Historia Natural; de Antiguedades y Arquitectura Militar, que son muy curiosas todas.

Ninguna ciudad de Italia posée, tal vez, un cementerio tan hermoso como el de bolonia: dividido en muchas partes, según los grados del cuerpo social, se hace observar por una multitud de monumentos y mausoleos ya sean pintados, ya esculpidos que, excitando la curiosidad conservan la memoria de sus ilustres difuntos, e imponen, el mismo tiempo, un sentimiento de veneración. A pesar de ser muy distante de la Ciudad, comunica con esta con un hermoso pórtico, el que, alargándose más y más, conduce al Santuario de la Madona de S. Luca cuya vista es magnífica, descubriéndose desde este punto la fertil llanura de la Lombardía. Tiene el pórtico hasta el Santuario, legua y media de extensión.

Es Bolonia, la residencia de dos Cardenales, el uno para lo Civil y el otro para lo Eclesiástico, el que es también Archicancelario de la Universidad. 
Las bolonianas, van, en todo, como las francesas.

Me había olvidado de hacer un elógio del clíma de Bolonia. Este es insoportable. Un aire glacial, venido de los Apeninos, que están al Norte de dicha ciudad helaba todos nuestros miembros, particularmente, piés y manos. Hubo día que estuvo el termómetro a 6 y 7 grados bajo cero. Túvele por compañero inseparable todo el tiempo que estuve en la Universidad haciendo mi oración.

\section{GASTO}

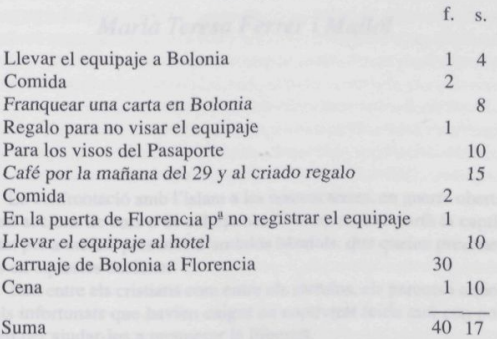

Nota.- Cada duro francés pierde en Toscana unos seis o siete sueldos, pués dan, de cada uno de ellos, 8 paulos y seis gracias. 